\title{
Three-dimensional rotation of even-even triaxial nuclei
}

\author{
Makito O* and Philip M. Walker \\ Department of Physics, University of Surrey, Guildford, Surrey GU2 7XH, United Kingdom
}

\begin{abstract}
With the self-consistent three-dimensional cranked Hartree-Fock-Bogoliubov (3d-cranked HFB) method, various types of rotational motion near the yrast line are investigated in an even-even nucleus in the $A \simeq 130$ mass region $\left({ }_{58}^{134} \mathrm{Ce}_{76}\right)$. The possibilities of chiral rotations, tilted-rotations, and dynamical aspects of these rotations are discussed through the analysis of the $3 \mathrm{~d}$-cranked HFB solutions. Although a stable planar solution of the chiral rotation is obtained, an aplanar chiral configuration is found to be unstable when triaxial deformation is treated self-consistently.
\end{abstract}

PACS numbers: 21.10.Re, 27.60.+j

Nuclei in the $A \simeq 130$ region are now of special interest because it is suggested that a new form of nuclear rotation can be expected: a chiral rotation [1, 2]. This threedimensional rotation was predicted at first in odd-odd systems, such as ${ }_{59}^{134} \mathrm{Pr}_{75}$, whose total angular momentum is built by a particle and a hole in valence orbitals in addition to a triaxial rotor, under an assumption of the irrotational-flow moment of inertia. Their angular momentum vectors point along the shortest $(s)$, longest $(l)$ and intermediate $(i)$ axes of the triaxially deformed nucleus $\left(\gamma \simeq 30^{\circ}\right)$ [17], respectively. When the collective angular momentum is absent, the total angular momentum consists only of the single-particle spins. The corresponding state is called "planar" because the total angular momentum is in the $s-l$ plane. The planar states correspond to "bandheads" of the chiral rotational bands. Rotational members built upon the planar state are classified as "aplanar" states because these states have the genuine chiral configuration. Possible experimental evidence of the nuclear chirality was reported in $N=75$ odd-odd isotones in the same mass region [3], through findings of pairs of near-degenerate $\Delta I=1$ bands (socalled "chiral doublets").

It is an interesting question whether or not eveneven nuclei can have the chiral configuration after breaking (Cooper) pairs. To realise the nuclear chirality in even-even systems, at least four quasi-particles must be excited; two neutrons and two protons. This multiquasiparticle excited state is expected at high spin where rotation-alignment occurs due to the Coriolis force. It is likely that such a "simultaneous" aligned state would be seen at higher spin, compared with the odd-odd chiral systems. Experimentally, a candidate for chiral doublets in even-even nuclei is suggested in ${ }_{60}^{136} \mathrm{Nd}_{76}$ [4], but there is as yet no detailed theoretical analysis about the possibility of chiral solutions in even-even nuclei, except a brief conference report [5].

The original model of the nuclear chirality is based on the particle(hole)-plus-rotor model which admits the presence of a macroscopic rotor with solid triaxial defor-

*Electronic address: m.oi@surrey.ac.uk mation $\left(\gamma \simeq 30^{\circ}\right)$. Although there is no concept of a rotor in fully microscopic models like the HFB approach, the rotor spin can be treated as the collective part of angular momentum in the cranking model, which can handle the collective and single-particle angular momentum on the same footing. An analysis of the chiral configuration in odd-odd systems was carried out through the Tilted-Axis Cranking model (TAC) 2]. Although each microscopic calculation (TAC) is made for given parameters of shape and pairing gaps as well as rotational frequency, these parameters are carefully determined by a macroscopic + microscopic approach (the Strutinski method) in terms of the energy minimisation.

If nuclear chirality is produced in even-even systems by four or more quasiparticle excitations, where the quasiparticles occupy orbitals with very different orientation, it is expected that the collective and individual (quasi)particle motions would be strongly coupled through the Coriolis force. Besides, the degree of triaxial deformation can be susceptible to the influence coming from rotationalignments at high spin, especially in those nuclei whose Fermi level is in the upper shell (i.e., high- $\Omega$ Nilsson orbits) [6, 7]. Therefore, if we attempt to describe the above physical situations, purely microscopic and selfconsistent treatments should be made with respect to nuclear shape and pairing. In other words, for the aim of demonstrating the chirality in even-even systems, it is necessary to show the emergence of a genuine threedimensional rotation together with the presence of substantial triaxial deformation. Furthermore, compared to odd-odd systems, it is numerically advantageous for us to study even-even systems because the treatment of pairing is more straightforward.

In this paper, we report a fully microscopic and selfconsistent study of three-dimensional nuclear rotations in even-even nuclei in the $A \simeq 130$ mass region near the yrast line [18], by means of the three-dimensional cranked Hartree-Fock-Bogoliubov method (3d-cranked HFB) [8, 9].

First of all, let us review the method. The HFB equation is self-consistently solved with the pairing-plus$\mathrm{Q} \cdot \mathrm{Q}$ force under constraints on particle numbers (proton and neutron), and angular momentum (length and orientation). There are six conditions relevant to the 
latter constraints. They are, $\left\langle\hat{J}_{1}\right\rangle=J \cos \theta,\left\langle\hat{J}_{2}\right\rangle=$ $J \sin \theta \sin \phi,\left\langle\hat{J}_{3}\right\rangle=J \sin \theta \cos \phi$, and $\left\langle B_{i}\right\rangle=0(i=$ $1,2,3)$, with $B_{i} \equiv \frac{1}{2}\left(\hat{Q}_{j k}+\hat{Q}_{k j}\right)(i, j, k$ : cyclic and each index runs from 1 to 3 ). The last three conditions are necessary when self-consistent calculations are performed in shape, so as to fix the intrinsic coordinate system set along the principal axes of the quadrupole tensor at rest (i.e., $J=0)$ 9]. Note that azimuthal $(\phi)$ and polar $(\theta)$ angles are measured, respectively, from the 1-3 plane and the 1 -axis. The nucleus ${ }_{58}^{134} \mathrm{Ce}_{76}$ is chosen for this study because of the following three reasons: (i) it is in the $A \simeq 130$ region where chiral doublets are seen, (ii) it is known to be $\gamma$-soft in its ground state [10], and (iii) it has its Fermi levels for protons and neutrons located in the high- $j$ intruder orbitals $\left(\mathrm{h}_{11 / 2}\right)$ with low- and high$\Omega$ components, respectively. According to Baranger and Kumar 11], two major shells of the spherical Nilsson basis are needed for each sector of isospin $(\mathrm{N}=4$ and 5 , for proton and neutron), in addition to intruder orbits $\left(i_{13 / 2}\right.$ both for proton and neutron). The force strengths for the quadrupole and pairing parts are determined through the (no-cranking) Nilsson + BCS model with the deformation parameters $(\beta, \gamma)=\left(0.162,0.0^{\circ}\right)$ and gap energies $\left(\Delta_{p}, \Delta_{n}\right)=(1.069,0.881)[\mathrm{MeV}][13]$. The corresponding wave function is used as an initial state for the self-consistent calculations at $J=0$. The HFB equation for an arbitrary spin $(J)$ is consecutively solved by means of the method of steepest descent 14] through the principal-axis cranking procedure $\left(\theta=0^{\circ}\right)$ with a step $\Delta J=0.1 \hbar$ until high spin where a four quasi-particle state appears with significant triaxial deformation. The pairing gaps and quadrupole deformation parameters are calculated in a fully self-consistent manner. For instance, they are calculated to be $(\beta, \gamma)=\left(0.132,25^{\circ}\right)$ and $\left(\Delta_{p}, \Delta_{n}\right)=(0.00,0.18)[\mathrm{MeV}]$ at $J=30 \hbar$. The self-consistent tilted-axis cranking calculations for $J$ start with this principal-axis cranked HFB state as the initial state. We confirmed that, similarly to the principal-axis cranking, the steepest descent method may be employed to solve the HFB equation for given $\phi$ along the direction of $\theta$ from $0^{\circ}$ to $90^{\circ}$ with a step $\Delta \theta=0.5^{\circ}$. $\phi$ is given every $1^{\circ}$ from $0^{\circ}$ to $90^{\circ}$. In this way, physical quantities, such as the energy surface $E^{J}(\phi \theta)$, quadrupole deformations $\beta^{J}(\phi \theta), \gamma^{J}(\phi \theta)$ and pairing gaps $\Delta_{\tau}^{J}(\phi \theta)(\tau=\mathrm{p}, \mathrm{n})$, are calculated for the given spin $J$ as a function of tilt angles $(\theta, \phi)$.

At first, let us see the energy surface at $J=26 \hbar$ (Fig (1). There is a minimum at $(\theta, \phi) \simeq\left(90^{\circ}, 45^{\circ}\right)$. Deformations are calculated to be $(\beta, \gamma)=\left(0.16,17^{\circ}\right)$. Major components of the single-particle spins come from both proton and neutron $\mathrm{h}_{11 / 2}$ orbitals, and they are respectively calculated as $\left(j_{1}, j_{2}, j_{3}\right)=(0.0,9.3,2.0)$ and $(0.2,2.5,9.6)$. (The unit for angular momentum is $\hbar$ here and hereafter.) The 1-, 2-, and 3-axes correspond to the $i-, s-$, and $l$-axes, respectively, in accordance with the $\gamma$ value. The proton and neutron $\mathrm{h}_{11 / 2}$ orbitals thus point along the $s-$ and $l$-axes, respectively. Col- lective rotation around the $i$-axis turns out not to be present. This situation implies that the corresponding states are the "planar" solutions of the chiral configuration. (There is another minimum at $(\theta, \phi) \simeq\left(47^{\circ}, 0^{\circ}\right)$, but this state is identical to the minimum we have just discussed above, from the analysis of the single-particle configuration, shape parameters, etc.)

The "aplanar" chiral configuration would be expected at $J=28 \hbar$ if collective motion could be added around the $i$-axis to the planar states in an "adiabatic" manner. Adiabaticity here means the same as in the context of the discussion of rotational bands such as the ground state rotational bands (g-bands). Analyses of the shape parameters, and of single-particle angular momenta for $\mathrm{h}_{11 / 2}$ protons and neutrons over all the energy surface imply that the chiral configuration [19] is realised for $(\theta, \phi) \simeq\left(70^{\circ}, 45^{\circ}\right)$ at $J=28 \hbar$. The corresponding quadrupole deformations are $(\beta, \gamma)=\left(0.13,32^{\circ}\right)$, and the alignments for proton and neutron $\mathrm{h}_{11 / 2}$ orbitals are $(1.9$, $9.3,2.4)$ and $(3.7,4.3,11.5)$, respectively. However, as seen in Fig 2 this state is near a saddle point, implying that this chiral configuration is unstable. The planar configuration, that forms an energy minimum at $J=26 \hbar$, is quite susceptible to the collective rotation, through the Coriolis force.

It is interesting to see what happens when the angular momentum is further increased to $J=30 \hbar$. Fig 3 shows the energy surface at $J=30 \hbar$. We have confirmed that similar structures to this energy surface are seen up to $J \simeq 38 \hbar$. There are six shallow minima found in the surface at $J=30 \hbar$, including an extremely shallow one. Let us label these minima as follows: $\mathcal{A}$ : $(\theta, \phi) \simeq\left(58^{\circ}, 42^{\circ}\right)$, $\mathcal{B}_{1}:(\theta, \phi) \simeq\left(28^{\circ}, 90^{\circ}\right), \mathcal{B}_{2}:(\theta, \phi) \simeq\left(90^{\circ}, 25^{\circ}\right), \mathcal{C}_{1}:$ $\theta \simeq 0^{\circ}, \mathcal{C}_{2}:(\theta, \phi) \simeq\left(90^{\circ}, 0^{\circ}\right), \mathcal{C}_{3}:(\theta, \phi) \simeq\left(90^{\circ}, 90^{\circ}\right)$. The groups $\mathcal{A}, \mathcal{B}$ and $\mathcal{C}$ represent a three-dimensional rotating solution (which we call "3d-rotation"), twodimensional solutions ("tilted axis rotation", or "TAR"), and one-dimensional solutions ("principal axis rotation", or "PAR"), respectively. Let us discuss these minima one by one.

The minima $\mathcal{C}_{i}(i=1,2,3)$ : This group is classified as one-dimensional rotation, or PAR. The minima are located at $(\theta, \phi) \simeq\left(0^{\circ},-\right),\left(90^{\circ}, 0^{\circ}\right)$, and $\left(90^{\circ}, 90^{\circ}\right)$ for $\mathcal{C}_{1}$, $\mathcal{C}_{2}$, and $\mathcal{C}_{3}$, respectively. The $\beta$ deformation values for these minima are almost equal $(\beta \simeq 0.13)$. The dominant components of total angular momentum come from proton and neutron $\mathrm{h}_{11 / 2}$ orbitals in either case. The $\gamma$ deformation values are about $25^{\circ}, 30^{\circ}$, and $-31^{\circ}$ for $\mathcal{C}_{1}$, $\mathcal{C}_{2}$, and $\mathcal{C}_{3}$, respectively, so that all of them are strongly triaxially deformed. $\mathcal{C}_{1}$ and $\mathcal{C}_{2}$ are identical from the analyses of their single-particle configurations, energy, deformation, etc. They show a rotation around the $i$ axis while $\mathcal{C}_{3}$ shows a rotation around the $s$-axis. These two types of one-dimensional rotation can be classified by analogy with classical mechanics. The moment of inertia for a rigid body $\left(\mathcal{J}^{\text {rig }}\right)$ and irrotational flow $\left(\mathcal{J}^{\text {irr }}\right)$ are then given as $\mathcal{J}_{n}^{\text {rig }} \propto 1-\sqrt{\frac{5}{4 \pi}} \beta \cos \left(\gamma-\frac{2 \pi}{3} n\right)$ and 
$\mathcal{J}_{n}^{\text {irr }} \propto \beta^{2} \sin ^{2}\left(\gamma-\frac{2 \pi}{3} n\right)$, respectively $(n=1,2,3)$. At $\gamma=30^{\circ}$, the $i$-component of the moment of inertia becomes largest for the irrotational flow, while it is the $s$-component for the rigid body. $\mathcal{C}_{1}$ is thus like "irrotational flow" and $\mathcal{C}_{3}$ is like "rigid body" rotation. These two types are energetically degenerate in the present case, so that the irrotational and rigid-body characters coexist in this high-spin state.

The minima $\mathcal{B}_{i}(i=1,2)$ : The attribute of this group is two-dimensional rotation, or TAR. $\mathcal{B}_{1}$ at $(\theta, \phi) \simeq$ $\left(28^{\circ}, 90^{\circ}\right)$ and $\mathcal{B}_{2}$ at $\left(90^{\circ}, 25^{\circ}\right)$ are likely to be the same states (that is, just a rearrangement of the principal axes). Their deformations are calculated to be $(\beta, \gamma) \simeq$ $\left(0.13,29^{\circ}\right)$. The $1-, 2$ - and 3 -axes correspond to $i-, s-$ and $l$-axes, respectively. Again proton and neutron $\mathrm{h}_{11 / 2}$ orbitals play a leading role in building total angular momentum. Their components are $(6.2,7.0,0.0)$ for protons and $(11.2,3.8,0.0)$ for neutrons. The neutron $h_{11 / 2}$ orbits have substantial components along the $i$-axis, making the total angular momentum vector for $\mathcal{B}_{i}$ lie in the $s-i$ plane. This configuration is different from the "planar" chiral configuration having the total angular momentum vector in the $s-l$ plane. The energy surface around $\mathcal{B}$ is shallow along one direction, for example, along $\phi$ for $\mathcal{B}_{2}$. This structure implies the possibility of fluctuation around the TAR.

The minimum $\mathcal{A},(\theta, \phi) \simeq\left(58^{\circ}, 42^{\circ}\right)$ : From the tilt angles, this "minimum" seems to correspond to the state with 3d-rotation, but in fact this is not a 3d-rotating state. The quadrupole deformations are calculated to be $(\beta, \gamma)=\left(0.12,60^{\circ}\right)$, so that the corresponding nuclear shape is oblate, that is, axially symmetric rather than triaxial. The major contribution to the total angular momentum comes from the proton and neutron $\mathrm{h}_{11 / 2}$ orbits. They are $(4.8,8.3,1.4)$ for protons, and $(10.1,4.0,6.7)$ for neutrons. In this case, the shortest axis (i.e., the symmetry axis) corresponds to the 2-axis. The alignment along the $s$-axis comes predominantly from the proton $\mathrm{h}_{11 / 2}$ component, which is consistent with the chiral configuration in odd-odd systems. However, the neutron $\mathrm{h}_{11 / 2}$ component contributes to building angular momentum along both the 1- and 3-axes. This is because the principal axes of the quadrupole tensor along both of the coordinates are identical, due to the axial symmetry around the $s$-axis. Therefore, this state is not like the chiral rotation, but rather corresponds to TAR of an oblate deformed nucleus. This minimum $\mathcal{A}$ is extremely shallow (depth is of the order of $10 \mathrm{keV}$ ), so that the adjacent states are energetically degenerate, implying the presence of a "zero mode". Such a dynamical motion will be discussed later.

The group $\mathcal{B}$ contains the lowest minima (hence the possible "yrast" state) and the group $\mathcal{C}$ has the second lowest $\left(E_{\text {ex }} \simeq 10 \mathrm{keV}\right.$ with respect to the energy of $\left.\mathcal{B}_{i}\right)$. At the minimum $\mathcal{A}, E_{\text {ex }} \simeq 300 \mathrm{keV}$. As is easily seen from the energy surfaces being very shallow, it is more natural to think that these states are coupled dynamically [20]. Namely, the state observed in reality will be a superposition of these minima. Nevertheless, it is useful and meaningful physically to classify these dynamical states in terms of several simpler modes. Let us now discuss the dynamical aspects of these minima.

First of all, a fluctuation around $\mathcal{B}_{i}$ should be studied because they are the lowest minima. The potential-well, for instance, around $\mathcal{B}_{2}$ extends one-dimensionally toward the direction of $\phi$, indicating a swing motion around TAR. This dynamical motion can be called "nutation". The nutation is likely to occur for $|\phi| \lesssim 40^{\circ}$ with $\theta=90^{\circ}$ in the case of $\mathcal{B}_{2}$. The signature symmetry, a symmetry with respect to $\pi$-rotation around one of the principal axes, say the $i$-axis, is broken in TAR. However, the nutation plays a role in restoring the symmetry dynamically, as seen in the multi-band crossings in the $A \simeq 180$ region [15]. Another oscillatory motion is expected also with respect to $\theta$, although this degree of freedom can be treated as a minor correction to the nutation. For these dynamical states, the quadrupole deformations are calculated to be almost constant and decoupled from the tilting degrees of freedom.

The second type of dynamical motion is expected around the minimum $\mathcal{A}$ along the shallow plateau. Because of the oblate deformation, all the states rotated around the $s$-axis are energetically degenerate. This sort of collective rotation is regarded as a zero mode, which is closely connected to the restoration of a continuous symmetry. In this case, the axial symmetry broken by the TAR of an oblate deformed state is restored by this zero mode. Noting the convention of the tilt angles, i.e, $\left(J_{1} / J, J_{3} / J\right)=(\cos \theta, \sin \theta \cos \phi)$, we rotate the state $\mathcal{A}$ around the $s$-axis (corresponding to the 2 -axis in this case) by $\alpha$. The rotated state is then expressed as $\theta(\alpha)=\arccos \left(J_{1}^{\mathcal{A}} \cos \alpha / J-J_{3}^{\mathcal{A}} \sin \alpha / J\right)$ and $\phi(\alpha)=\arccos \left\{\csc \theta(\alpha)\left(J_{1}^{\mathcal{A}} \sin \alpha / J+J_{3}^{\mathcal{A}} \cos \alpha / J\right)\right\}$. Here, $\left(J_{1}^{\mathcal{A}}, J_{2}^{\mathcal{A}}, J_{3}^{\mathcal{A}}\right)$ denotes the components of total angular momentum for the minimum $\mathcal{A}$. The trajectory $(\theta(\alpha), \phi(\alpha))$ is drawn in Fig 3 showing that the shallow plateau near $\mathcal{A}$ corresponds to the zero mode rotation. Although the oblate deformed solution collapses beyond the plateau, the structure of the energy surface between the minima $\mathcal{B}_{i}$ seems to retain the influence from this zero mode. If the tunnelling between these minima through $\mathcal{A}$ has a physical meaning, it should be related to the restoration of the broken symmetry, as previously discussed by Bonche et al. [16].

These dynamical modes are related to restoration of symmetries. In the first case, the nutation of the triaxialdeformed state restores the discrete signature symmetry, while the zero mode of the oblate-deformed TAR state restores the continuous axial symmetry in the second. The explicit treatment of these modes should be made by means of the generator coordinate method, which it is planned to achieve in the future.

In summary, the self-consistent $3 \mathrm{~d}$-cranked HFB equation is solved for a $\gamma$-soft nucleus ${ }^{134} \mathrm{Ce}$, and several solutions are obtained for one and two-dimensional rotations (PAR, TAR, and planar). All the solutions are built upon 
four quasi-particle states of $\mathrm{h}_{11 / 2}$ orbitals for protons and neutrons. The chiral configuration is found as a planar state (i.e., the "bandhead" of the chiral rotational band), but its aplanar partner turns out to be unstable in the present case. As the angular momentum increases, the corresponding configuration evolves into an oblate deformed state whose rotational motion is TAR-like. This oblate state is seen over a relatively wide range of angular momentum at high $\operatorname{spin}(30 \hbar \lesssim J \lesssim 38 \hbar)$. As a general characteristic, it seems that the rotational motion of ${ }^{134} \mathrm{Ce}$ at high spin tends to be dynamical and nonuniform (nutation). Furthermore, it should be noted that the chiral solutions in odd-odd systems are also in quite shallow minima in the energy surface (barrier heights range from 50 to $100 \mathrm{keV}$ ) [2, 5]. However, there is an argument in the framework of the particle-rotor model that the tunnelling between chiral minima is suppressed when the mass parameter is large [5]. More studies may be necessary to understand the dynamical aspects of nuclear chirality, particularly in the context of the coupling between deformation and orientation degrees of freedom, in theory and hopefully in experiment.

M.O. thanks Professor H. Flocard for discussions that motivated us to study nuclear chirality. He is also grateful to Professor P.-H. Heenen for fruitful discussions and the information of Ref. [16]. Our appreciation to Dr. P.H. Regan is acknowledged for the information on Ref. 4]. This work is supported by an EPSRC advanced research fellowship GR/R75557/01.
[1] S. Frauendorf, and J. Meng, Nucl. Phys. A617, 131 (1997);

[2] V. I. Dimitrov, S. Frauendorf, and F. Dönau, Phys. Rev. Lett. 84, 5732 (2000).

[3] K. Starosta, et al., Phys. Rev. Lett. 86, 971 (2001).

[4] E. Mergel, et al., Eur. Phys. J. A 15, 417 (2002).

[5] V. Dimitrov, F.Dönau, and S. Frauendorf, international conference "Frontiers of Nuclear Structure" (Berkeley, 2002); nucl-th/0211063.

[6] R. R. Hilton, H. J. Mang, and P. Ring, Phys. Rev. Lett. 43, 1979 (1979).

[7] M. Oi, P. M. Walker, and A. Ansari, Phys. Lett. B505, $75,(2001)$.

[8] T. Horibata and N. Onishi, Nucl. Phys. A596, 251 (1996).

[9] A. K. Kerman, and N. Onishi, Nucl. Phys. A361, 179 (1981).

[10] N. J. O'Brien, et al., Phys. Rev. C59, 1334 (1999).

[11] M. Baranger, and K. Kumar, Nucl. Phys., 62, 113 (1965); K. Kumar, M. Baranger, Nucl. Phys. A110, 529 (1968).
[12] P. Ring and P. Schuck, Nuclear Many-body Problem (Springer-Verlarg, Berlin, 1980) p.8.

[13] P. Möller, J. R. Nix, W. D. Myers, and W. J. Swiatecki, Atomic Data Nucl. Data Tables 59, 185 (1995).

[14] N. Onishi, Nucl. Phys. A456, 279 (1986).

[15] M. Oi, A. Ansari, T. Horibata, and N. Onishi, Phys. Lett. B 480, 35 (2000).

[16] P. Bonche, J. Dobaczewski, H. Flocard, and P.-H. Heenen, Nucl. Phys. A 530, 149 (1991).

[17] The Hill-Wheeler parameterization [12] is employed for the quadrupole deformation parameters, $\beta$ and $\gamma$, where the sign of $\gamma$ is opposite to the Lund convention.

[18] States with highest spin for given energy.

[19] Note that a "chiral configuration" does not necessarily mean a "chiral minimum" or "chiral solution".

[20] There are arguments that the large mass parameter can suppress the dynamical motions. A brief discussion will be given later in this paper. 


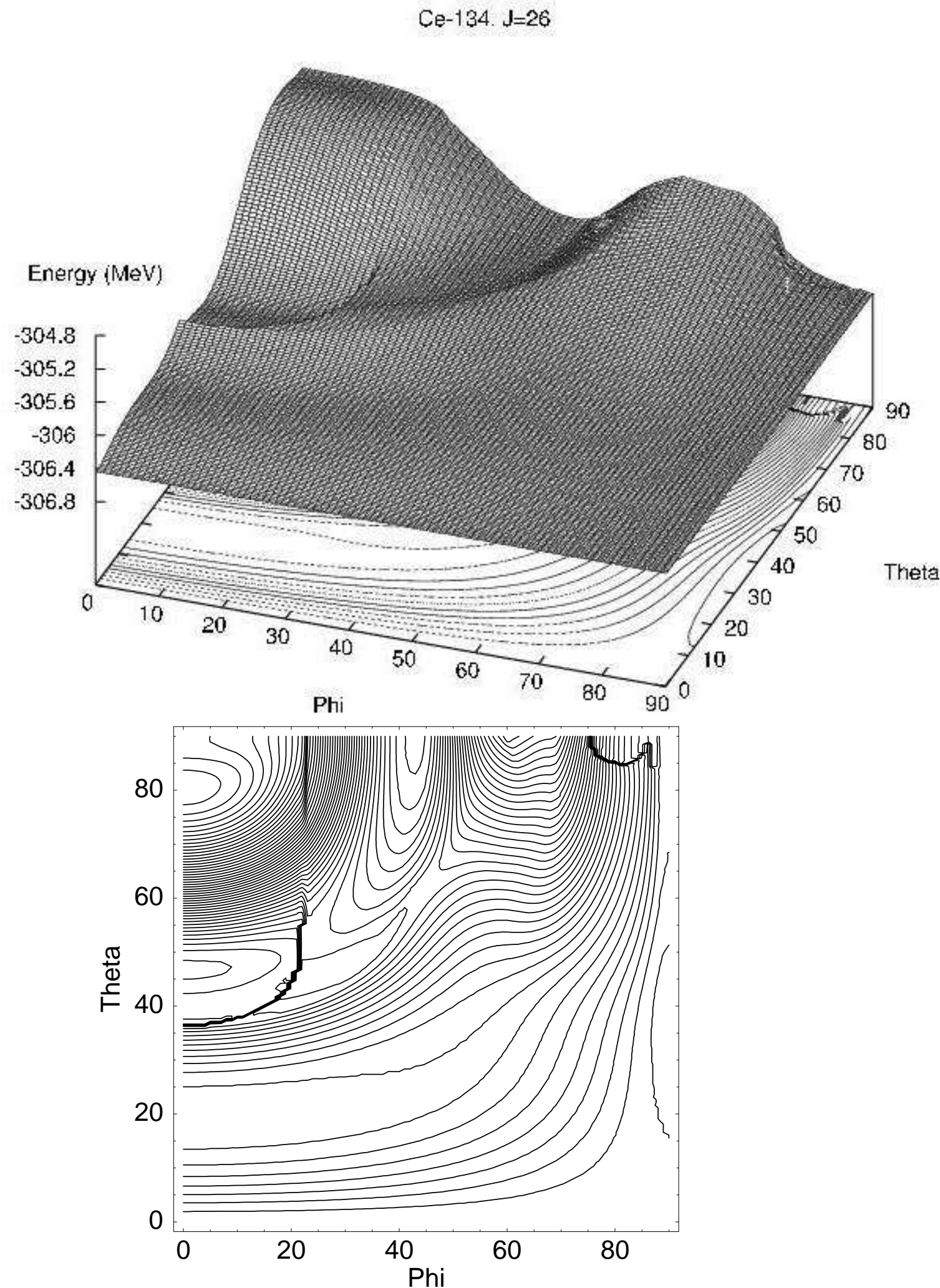

FIG. 1: Energy surface at $J=26 \hbar$. The contour interval in the lower panel is $50 \mathrm{keV}$. Discontinuities in the energy surface are due to configuration changes, or level crossings. 


\section{Ce-134. $J=28$}

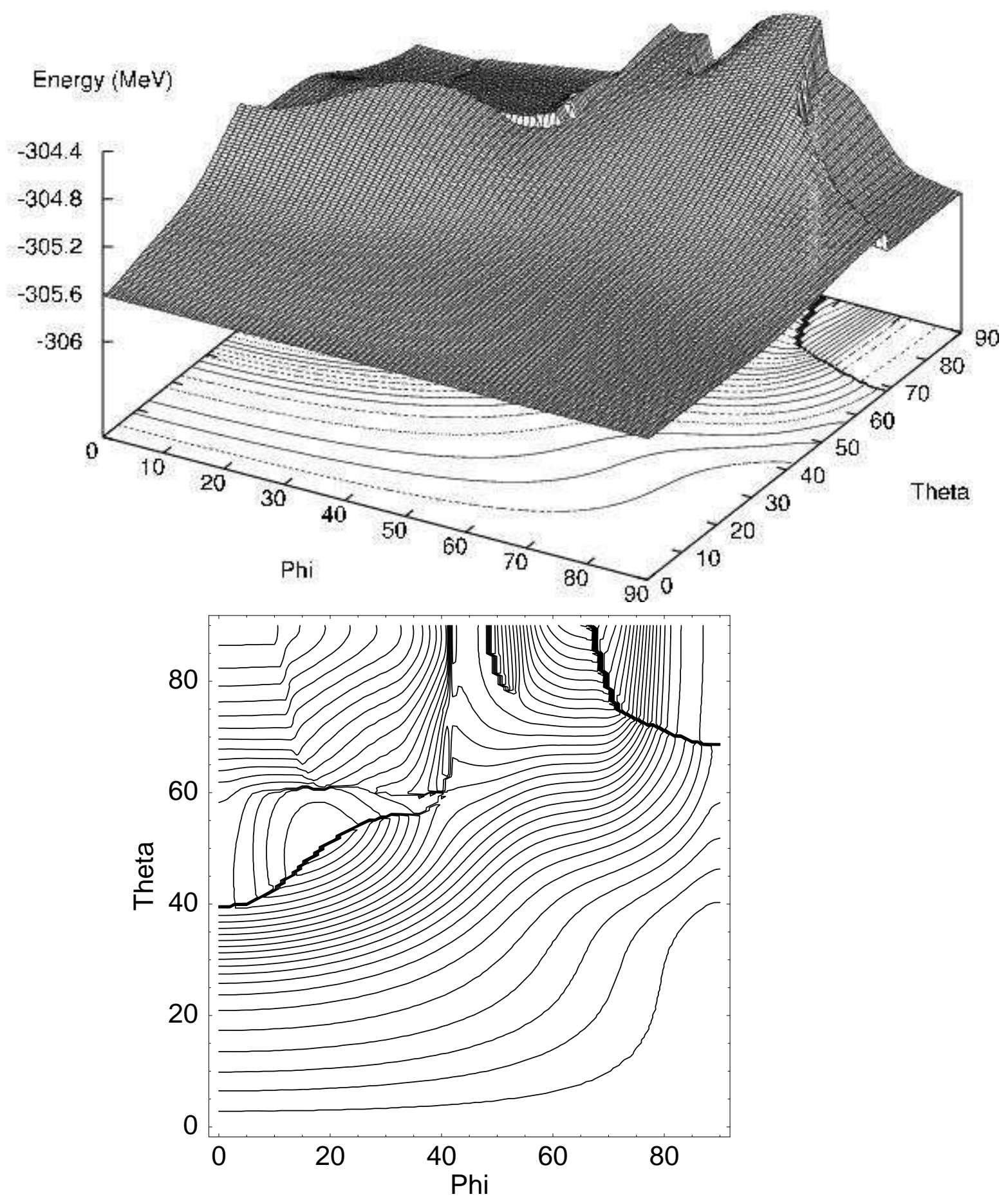

FIG. 2: Energy surface at $J=28 \hbar$. The contour interval in the lower panel is $40 \mathrm{keV}$. 
Ce-134. J $=30$

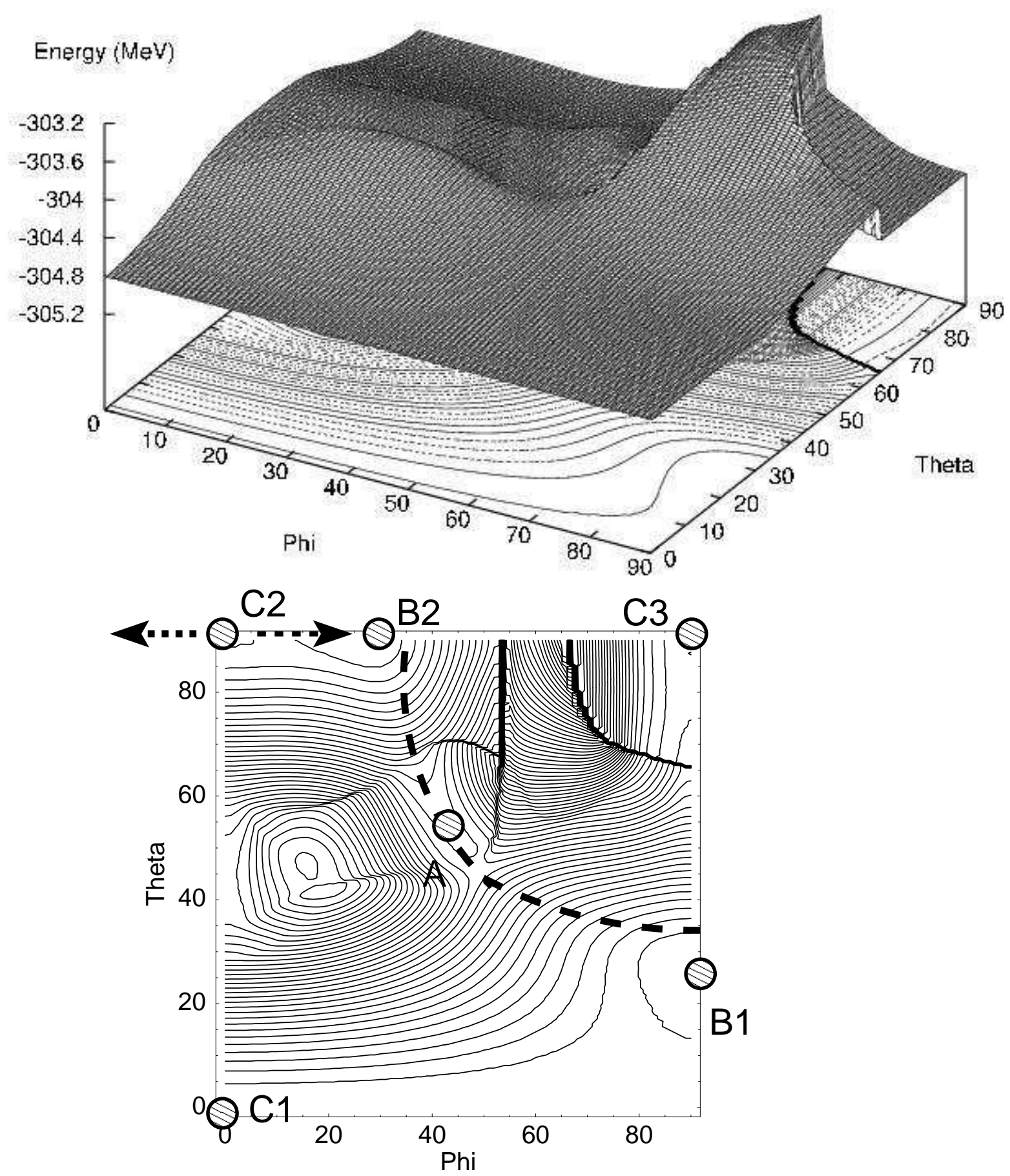

FIG. 3: Energy surface at $J=30 \hbar$. The contour interval in the lower panel is $20 \mathrm{keV}$. The trajectory of the zero mode of the oblate state $\mathcal{A}$ is schematically displayed by the dashed curve without an arrow. The straight line with arrows indicates the nutation. 